https://doi.org/10.15407/ujpe64.11.1059

R.M. PLYATSKO, M.T. FENYK

Ya. S. Pidstryhach Institute for Applied Problems of Mechanics and Mathematics,

Nat. Acad. of Sci. of Ukraine

(3b, Naukova Str., Lviv 79060,Ukraine; e-mail: plyatskor@gmail.com)

\title{
ON REACTION OF A SPINNING PARTICLE ON THE SPACETIME CURVATURE ${ }^{1}$
}

\begin{abstract}
The reaction of a classical (nonquantum) spinning particle on the spacetime curvature according to the Mathisson-Papapetrou equations is analyzed. From the point of view of the observer comoving with the particle in Schwarzschild's field, this reaction is a reaction on the gravitomagnetic components of the gravitational field. The values of these components significantly depend on the relativistic Lorentz factor calculated by the particle velocity relative to the Schwarzschild mass. As a result, the value of the spinning particle acceleration relative to the geodesic motion is proportional to the second power of the Lorentz factor. At the same time, the intensity of the electromagnetic radiation of a charged spinning particle is proportional to the fourth power of this factor. Some numerical estimates are presented.
\end{abstract}

Keywords: spinning particle, Mathisson-Papapetrou equations, Schwarzschild's field, strong spin-gravity coupling.

\section{Introduction}

In general relativity, two approaches have been developed for the description of the spinning particle behavior in a gravitational field. Chronologically, the first one was initiated by V. Fock, D. Ivanenko, and H. Weyl in 1929, when the usual Dirac equation was generalized for a curved spacetime [1-3]. The second classical (nonquantum) approach was proposed in 1937 by M. Mathisson [4]. Much later it was shown that, in a certain sense, the classical equations follow from the general relativistic Dirac equation as a classical approximation [5, 6]. After M. Mathisson, the same equations for a macroscopic spinning test body (particle) were derived by A. Papapetrou by another method [7]. Therefore, these equations are known now as the Mathisson-Papapetrou (MP) equations.

In the focus of this article are the MP equations as an important source of knowledge about the reaction of a spinning particle on the spacetime curvature which manifests itself in the properties of the particle motion in Schwarzschild's background. In Sec. 2, the MP equations and some their consequences are presented. The dependence of the spin-gravity coupling on the particle velocity is considered in Sec. 3 . The estimation of the intensity of the electromagnetic ra-

(C) R.M. PLYATSKO, M.T. FENYK, 2019

ISSN 2071-0194. Ukr. J. Phys. 2019. Vol. 64, No. 11 diation for a charged spinning particle moving with ultrarelativistic velocity is given in Sec. 4. Some numerical estimates for electrons, protons, and neutrinos are presented in Sec. 5. We conclude in Sec. 6.

\section{Mathisson-Papapetrou Equations}

The initial form of the MP equations is [4]

$$
\begin{aligned}
& \frac{D}{d s}\left(m u^{\lambda}+u_{\mu} \frac{D S^{\lambda \mu}}{d s}\right)=-\frac{1}{2} u^{\pi} S^{\rho \sigma} R_{\pi \rho \sigma}^{\lambda}, \\
& \frac{D S^{\mu \nu}}{d s}+u^{\mu} u_{\sigma} \frac{D S^{\nu \sigma}}{d s}-u^{\nu} u_{\sigma} \frac{D S^{\mu \sigma}}{d s}=0 \\
& S^{\lambda \nu} u_{\nu}=0
\end{aligned}
$$

where $u^{\lambda} \equiv d x^{\lambda} / d s$ is particle's 4 -velocity, $S^{\mu \nu}$ is the antisymmetric tensor of spin, $m$ and $D / d s$ are the mass and the covariant derivative along $u^{\lambda}$, respectively. Here and in the following, greek indices run through 1, 2, 3, 4, and latin indices run through 1 , 2,3 ; the signature of the metric $(-,-,-,+)$ and the unites $c=G=1$ are chosen. Relation (3) plays the role of a supplementary condition for Eqs. (1), (2), and its physical meaning lay in choosing the center of

1 This work is based on the results presented at the XI BolyaiGauss-Lobachevskii (BGL-2019) Conference: Non-Euclidean, Noncommutative Geometry and Quantum Physics. 
mass of a spinning particle. Often instead of (3), the relation

$S^{\lambda \nu} P_{\nu}=0$

where $P_{\nu}$ is the particle 4-momentum, is used. In the linear spin approximation, $P_{\nu}$ is proportional to $u_{\nu}$.

Equations (1)-(3) have the constant of motion

$S_{0}^{2}=\frac{1}{2} S_{\mu \nu} S^{\mu \nu}$,

where $\left|S_{0}\right|$ is the absolute value of the spin. The physical condition for a spinning test particle

$\frac{\left|S_{0}\right|}{m r} \equiv \varepsilon \ll 1$

must be taken into account [11], where $r$ is the characteristic length scale of the background space-time (in particular, for the Schwarzschild metric, $r$ is the radial coordinate).

It is important that the MP equations can be used for the investigation of spinning particle motions with any velocity relative to the source of a gravitational field (e.g., Schwarzschild's or Kerr's black hole) up to the speed of light, similarly as the geodesic equations are used for a fast moving spinless particle. The first effects of the spin-gravity interaction following from the MP equations in Schwarzschild's background were considered in [8]. According to this paper, the influence of spin on particle's trajectory is too small for a practical registration. The similar conclusion was stressed in the known book [9].

Nevertheless, another supposition can be find in [10]: "The simple act of endowing a black hole with angular momentum has led to an unexpected richness of possible physical phenomena. It seems appropriate to ask whether endowing the test body with intrinsic spin might not also lead to surprises". This paper together with [11], where the spin-spin and spin-orbit gravitational interactions were considered, gave the impulse for realizing the program of more detailed investigations of physical effects following from the MP equations without a priori restrictions on the influence of particle's spin on its trajectory. One of the first results of a realization of this program was presented in [12]. It was shown that specific situations in the spinning particle motions arise, when its becomes very close to the speed of light [12-14].

In the case where $S^{\mu \nu}=0$, Eqs. (1)-(3) reduce to the geodesic equations. In the linear spin approxima- tion, the term on the right-hand side of (1) determines the main contribution of particle's spin in the deviation of its motion from the geodesic one, i.e. the reaction of a particle on the spacetime curvature.

It is useful to consider the MP equations in their representation in terms of the local (tetrad) values, which correspond to the situation where an observer is comoving with the spinning particle. In the linear spin approximation, the MP equations yield the relation $[14,16]$

$\gamma_{(i)(4)(4)}=-\frac{S_{(1)}}{m} R_{(i)(4)(2)(3)}$,

where $\gamma_{(k)(1)(4)}$ are the Ricci coefficients of rotation, and the first local vector (1) is chosen here to be oriented along the particle's 3-vector of spin (this means that $\left.S_{(2)}=0, S_{(3)}=0\right)$. The value $\gamma_{(i)(4)(4)}$ is the dynamical characteristic of the reference frame, namely, its acceleration. According to (6), we have

$a_{(i)}=-\frac{S_{(1)}}{m} R_{(i)(4)(2)(3)}$,

where $a_{(i)}$ is the 3 -acceleration with which the spinning particle deviates from the free geodesic fall, as measured by the comoving observer. In the next section, we will consider expression (7) at different particle velocities relative to Schwarzschild's source of the gravitational field.

\section{Role of the Very High Particle Velocity}

To understand an important role of the spinning particle velocity in the spin-gravity coupling, let us consider the expression for the gravitomagnetic components $B_{(k)}^{(i)}$ of the gravitational field [15]

$B_{(k)}^{(i)}=-\frac{1}{2} R_{(m)(n)}^{(i)(4)} \varepsilon_{(k)}^{(m)(n)}$

(here, the parentheses note the local tetrad components of the corresponding values; and $\varepsilon_{(k)}^{(m)(n)}$ is the Levi-Civita symbol) in the partial case where the spinning particle is moving in the gravitational field of Schwarzschild's mass. We use the standard Schwarzschild coordinates $x^{1}=r, x^{2}=\theta, x^{3}=\varphi$, $x^{4}=t$, when the nonzero components of the metric tensor $g_{\mu \nu}$ are

$$
\begin{aligned}
& g_{11}=-\left(1-\frac{2 M}{r}\right)^{-1}, \quad g_{22}=-r^{2} \\
& g_{33}=-r^{2} \sin ^{2} \theta, \quad g_{44}=1-\frac{2 M}{r}
\end{aligned}
$$

ISSN 2071-0194. Ukr. J. Phys. 2019. Vol. 64, No. 11 
where $M$ is the mass of Schwarzschild's source of the gravitational field. We consider the case where the particle moves in the plane $\theta=\pi / 2$, and its spin [as well as the first space local axis (1)] is orthogonal to this plane. It is convenient to orient the second space axis (2) along the direction of particle's motion. Then we have

$$
\begin{aligned}
& B_{(2)}^{(1)}=B_{(1)}^{(2)}=\frac{3 M}{r^{3}} \frac{u_{\|} u_{\perp}}{\sqrt{\gamma^{2}-1}}\left(1-\frac{2 M}{r}\right)^{-1 / 2}, \\
& B_{(3)}^{(1)}=B_{(1)}^{(3)}=\frac{3 M}{r^{3}} \frac{u_{\perp}^{2} \gamma}{\sqrt{\gamma^{2}-1}},
\end{aligned}
$$

where $\gamma$ is the relativistic Lorentz factor of the moving particle, as estimated by an observer who is at rest relative to the source of a gravitational field. The value of $\gamma$ is given by the expression

$\gamma=\frac{1}{\sqrt{1-v^{2}}}$

where $v^{2}$ is the second power of particle's 3-velocity relative to the observer. In the case of the diagonal metric, according to the general expression for the 3 -velocity components $v^{i}$, we have

$v^{i}=\frac{d x^{i}}{\sqrt{g_{44}} d t}$.

For $v^{2}$, we write

$v^{2}=v_{i} v^{i}=\gamma_{i k} v^{i} v^{k}$,

where $\gamma_{i k}$ is the 3 -space metric tensor, with the following relation between $\gamma_{i k}$ and $g_{\mu \nu}$ for the diagonal metric: $\gamma_{i k}=-g_{i k}$. Relations (12)-(14) with $u_{\mu} u^{\mu}=1$ imply that

$\gamma=\sqrt{u_{4} u^{4}}$.

Let us compare the values from (10) and (11) at low and high velocities. When the velocity is low with $u_{\|}=\delta_{1}, u_{\perp}=\delta_{2},\left|\delta_{1}\right| \ll 1,\left|\delta_{2}\right| \ll 1$, and $\gamma^{2}-1=$ $\Delta^{2} \ll 1$, where

$\Delta^{2}=\left(1-\frac{2 M}{r}\right)^{-1} \delta_{1}^{2}+\delta_{2}^{2}$,

it follows from (10) and (11) that

$B_{(2)}^{(1)}=B_{(1)}^{(2)} \approx \frac{3 M}{r^{3}} \frac{\delta_{1} \delta_{2}}{\Delta}\left(1-\frac{2 M}{r}\right)^{-1 / 2}$,
$B_{(3)}^{(1)}=B_{(1)}^{(3)} \approx \frac{3 M}{r^{3}} \frac{\delta_{2}^{2}}{\Delta}$

In other words, at low velocities, the common term $3 M / r^{3}$ in the expressions for the gravitomagnetic components (17) and (18) is multiplied by corresponding small factors:

$\left|\frac{\delta_{1} \delta_{2}}{\Delta}\right| \ll 1, \quad\left|\frac{\delta_{2}^{2}}{\Delta}\right| \ll 1$

In the highly relativistic region, when $\gamma^{2} \gg 1$ and both $u_{\|}^{2}$ and $u_{\perp}^{2}$ have order $\gamma^{2}$, it follows from (10), (11) that

$B_{(2)}^{(1)}=B_{(1)}^{(2)} \sim \frac{3 M}{r^{3}}\left(1-\frac{2 M}{r}\right)^{-1 / 2} \gamma$,

$B_{(3)}^{(1)}=B_{(1)}^{(3)} \sim \frac{3 M}{r^{3}} \gamma^{2}$.

When only $u_{\perp}^{2} \gg 1$, with $u_{\|}^{2} \ll u_{\perp}^{2}$, the values from (19) are proportional to $u_{\|}$, and the values from (20) are proportional to $\gamma^{2}$. In the case where $u_{\|}^{2} \gg 1$ and $u_{\perp}^{2} \ll u_{\|}^{2}$, the values from (19) and (20) are proportional to $u_{\perp}$ and $u_{\perp}^{2}$, respectively.

Now, we take into account that, according to (7) and (8), just the gravitomagnetic components (8) determine the values of the acceleration $a_{(i)}$ from (7) by the expression

$a_{(i)}=-\frac{S_{(1)}}{m} B_{(i)}^{(1)}$.

Then, for the absolute value of the acceleration

$|\mathbf{a}|=\sqrt{a_{(1)}^{2}+a_{(2)}^{2}+a_{(3)}^{2}}$

in view of (19)-(21), we have

$|\mathbf{a}|=\frac{3 M}{r^{2}} \frac{\left|S_{0}\right|}{m r}\left|u_{\perp}\right| \sqrt{1+u_{\perp}^{2}}$,

and the vector $\mathbf{a}$ is oriented along the radial direction. According to (22), $|\mathbf{a}|$ does not depend on the radial component of the particle velocity and essentially depends on its tangential velocity. In the case of the highly relativistic motion with $u_{\perp}^{2} \gg 1$, relation (22) yields

$|\mathbf{a}|=\frac{3 M}{r^{2}} \varepsilon \gamma^{2}$,

where $\gamma$ is the Lorentz factor calculated by the tangential velocity $u_{\perp}$, and $\varepsilon$ is determined in (7). (Note 
that, in the above considered partial case of the particle motion in Schwarzschild's field, the relation $\left|S_{(1)}\right|=\left|S_{0}\right|$ is valid).

So, according to (23), the absolute value of the particle acceleration becomes much greater at highly relativistic velocities of the spinning particle than at low velocities. This means that the smallness of $\varepsilon$ from (5) does not lead to the conclusion about the small influence of the particle spin on its acceleration, as estimated by the comoving observer.

From (23), we get the qualitative criterion that indicates the value of $\gamma$ at which the reaction of a spinning particle on the spacetime curvature is significant. Indeed, if $\gamma$ is of order $1 / \sqrt{\varepsilon}$, the value of $|\mathbf{a}|$ is of order $M / r^{2}$ by (23) and is equal to the known value of the acceleration of a free fall in the Newtonian theory of gravity.

The results presented in this section describe the spin-gravity coupling in the proper frame of the spinning particle. At the same time, it is necessary to study the influence of the spin-gravity coupling on the particle trajectories, especially when its velocity is very high. Various cases of the essentially nongeodesic orbits of a highly relativistic spinning particle in Schwarzschild's field, which follows from the MP equations, are investigated in [16-21]. In particular, it is shown that, due to the strong action of the highly relativistic spin-gravity coupling, there are circular orbits of a spinning particle in the Schwarzschild field which significantly differ from the circular orbits of a spinless particle in this field. For a realization of these orbits, the spinning particle must possess the high velocity which corresponds to the value of $\gamma$ of the order $1 / \sqrt{\varepsilon}$.

\section{On Electromagnetic Radiation of a Charged Spinning Particle}

We use expression (23) to estimate the electromagnetic radiation of a spinning particle which possesses the electric charge $q$. Indeed, according to the known result of the classical electrodynamics, the intensity $I$ of the electromagnetic radiation in the frame, where the velocity of a charge particle is equal to 0 with nonzero acceleration $w$, is given by the expression

$I=\frac{2 q^{2} w^{2}}{3 c^{3}}$, where $c$ is the speed of light. Inserting expression (23) as $w$ in units with $c=1$ into (24), we get

$I=6 q^{2} \frac{M^{2}}{r^{4}} \varepsilon^{2} \gamma^{4}$

Equation (25) shows that, due the term $\gamma^{4}$, the value $I$ can be significant for some high tangential velocities even for small values of $\varepsilon$ and far from Schwarzschild's horizon $(r \gg 2 M)$.

\section{Numerical Estimates}

In addition to the small value of $\varepsilon$ from (5), it is convenient to consider the value of $\varepsilon_{0}$ determined by

$\varepsilon_{0} \equiv \frac{\left|S_{0}\right|}{m M}$

In contrast to $\varepsilon$, the value of $\varepsilon_{0}$ does not depend on the coordinate $r$. It is easy to check that, for an electron in the gravitational field of a black hole with three masses of the Sun, the value of $\varepsilon_{0}$ is equal to $4 \times 10^{-17}$. Then the necessary value of the $\gamma$-factor for a realization of some highly relativistic orbits by the electron near this black hole is of order $10^{8}$. This $\gamma$-factor corresponds to the energy of the electron free motion of order $10^{14} \mathrm{eV}$. Analogously, for a proton in the field of such black hole, the corresponding energy is of order $10^{18} \mathrm{eV}$. For the massive black hole, those values are greater: for example, if $M$ is equal to $10^{6}$ of Sun's mass, the corresponding value of the energy is of order $10^{17} \mathrm{eV}$ for an electron and is $10^{21} \mathrm{eV}$ for a proton. Naturally, far from the black hole, these values are greater, because the necessary $\gamma$-factor is proportional to $\sqrt{r}$.

Note that, for a neutrino near the black hole with three masses of the Sun, the necessary values of its $\gamma$-factor for motions on highly relativistic orbits correspond to neutrino's energy of a free motion of order $10^{5} \mathrm{eV}$. If black hole's mass is of order $10^{6}$ of Sun's mass, the corresponding value is of order $10^{8} \mathrm{eV}$.

\section{Conclusions}

It follows from the MP equations that, due to the reaction of a spinning particle on the spacetime curvature, its motion differs from the motion of a spinless particle. This deviation is small, when the velocity of a spinning particle relative to the Schwarzschild mass is not very high and becomes much greater at the velocities which are close to the speed of light. As a

ISSN 2071-0194. Ukr. J. Phys. 2019. Vol. 64, No. 11 
result, the ultrarelativistic charged spinning particle can generate the intense electromagnetic radiation, according to Eq. (25).

By the numerical estimates, one can suppose that the strong reaction of ultrarelativistic electrons and protons on the spacetime curvature can be manifest itself in their motions near some black holes, in particular, through the specific features of their electromagnetic radiation.

This work was supported by the budget program of Ukraine "Support for the development of priority research areas" (CPCEC 6451230).

1. V. Fock, D. Ivanenko. Über eine megliche geometrische Deutung der relativistischen Quantentheorie. Z. Phys. 54, 798 (1929).

2. V. Fock. Geometrisierung der Diracschen Theorie des Electrons. Z. Phys. 57, 261 (1929).

3. H. Weyl. Gravitation and the electron. Proc. Nat. Acad. Sci. USA 15, 323 (1929).

4. M. Mathisson. Neue Mechanik materieller Systeme. Acta Phys. Pol. 6, 218 (1937).

5. S. Wong. Heisenderg equations of motion for spin- $1 / 2$ wave equation in general relativity Int. J. Theor. Phys. 5, 221 (1972).

6. L. Kannenberg. Mean motion of Dirac electrons in a gravitational field. Ann. Phys. (N.Y.) 103, 64 (1977).

7. A. Papapetrou. Spinning test-particles in general relativity. Proc. R. Soc. A 209, 248 (1951).

8. E. Corinaldesi, A. Papapetrou. Spinning test-particles in general relativity. II. Proc. R. Soc. A 209, 259 (1951).

9. C.W. Misner, K.S. Thorne, J.A. Wheeler. Gravitation (Freeman, 1973).

10. S. Rasband. Black holes and spinning particles in general relativity. Phys. Rev. Lett. 30, 111 (1973).

11. R. Wald. Gravitational spin interaction. Phys. Rev. D 6, 406 (1972).

12. R.M. Plyatsko, A.L. Vynar. Essentially nongeodesic motions of a rotating test body in the general theory of relativity. Sov. Phys. Dokl. 27, 328 (1982).
13. R.M. Plyatsko. Manifestations of Gravitational Ultrarelativistic Spin-Orbit Interaction (Naukova Dumka, 1988) (in Ukrainian).

14. R. Plyatsko. Gravitational ultrarelativistic spin-orbit interaction and the weak equatorial principle. Phys. Rev. D 58, 084031 (1998).

15. K. Thorne, J. Hartle. Laws of motion and precession for black holes and other bodies. Phys. Rev. D 31, 1815 (1985).

16. R. Plyatsko, M. Fenyk, O. Stefanyshyn. In Equations of Motion in Relativistic Gravity 165 (Springer, 2015).

17. R. Plyatsko, M. Fenyk. Highly relativistic spin-gravity coupling for fermions. Phys. Rev. D 91, 064033 (2015).

18. R. Plyatsko, M. Fenyk. Reply to "Comment on 'Highly relativistic spin-gravity coupling for fermions' ". Phys. Rev. D 93, 028502 (2016).

19. R. Plyatsko, M. Fenyk. Antigravity: Spin-gravity coupling in action. Phys. Rev. D 94, 044047 (2016).

20. R. Plyatsko, M. Fenyk, V. Panat. Highly relativistic spingravity coupling. Phys. Rev. D 96 (2017) 064038.

21. R. Plyatsko, V. Panat, M. Fenyk. Nonequatorial circular orbits of spinning particles in the Schwarzschild-de Sitter background. Gen. Relativ. Gravit. 50, 150 (2018).

Received 29.08.19

Р.М. Пляцко, М.Т. Феник

ПРО РЕАКЦІЮ СПІНОВОЇ

ЧАСТИНКИ НА КРИВИНУ ПРОСТОРУ-ЧАСУ

$\mathrm{P}$ е $з$ ю м е

Проаналізовано реакцію класичної (неквантової) спінової частинки на кривину простору-часу згідно з рівняннями Матісона-Папапетру. 3 точки зору спостерігача, супутнього до частинки у полі Шварцшильда, вона визначається гравітомагнітними компонентами гравітаційного поля. Величини цих компонент суттєво залежать від релятивістського фактора Лоренца, обчисленого за швидкістю частинки відносно шварцшильдівської маси. Як наслідок, прискорення спінової частинки відносно геодезичного руху є пропорційним до квадрата фактора Лоренца. Водночас інтенсивність електромагнітного випромінювання зарядженої спінової частинки пропорційна до четвертого степеня цього фактора. 